\title{
The New Definitions of Death for Organ Donation: A Multidisciplinary Analysis from the Perspective of Christian Ethics by Doyen Nguyen
}

\author{
Peter Lang, 2018, paperback, \$145.90 \\ 590 pages, bibliographic references and index, ISBN 978-3-0343-3277-4
}

Doyen Nguyen argues that the criteria used to determine death for organ donationdeath by neurological criteria (DNC) or the circulatory criteria used in donation-after-circulatory-death (DCD) protocols-are invalid. The book is divided into three parts. Part 1 surveys the rationales for cadaveric organ procurement, arguing that they depend on new definitions of death. The history of brain death, the summary position of the Pontifical Academy for Life, and the writings of James Bernat are the departure points for her argument that DNC was invented to advance transplant medicine by way of the dubious assumption that the brain is the supreme integrator of the body. The criteria used in DCD protocols are then assumed to be valid because they indicate DNC. Part 2 consists of two chapters critiquing these definitions from Aristotelian, Thomistic, and contemporary perspectives. Catholic defenders of DNC are criticized for not sufficiently respecting the degree to which the brain-dead body can exhibit integrative unity, which should be taken to indicate the presence of the human soul. Part 3 consists of a single chapter arguing that the Church has not spoken authoritatively in favor of DNC and that current cadaveric procurement practices are incompatible with Christian ethics. On the whole, Nguyen marshals a thoroughgoing and lengthy study that will leave readers disturbed by the ambiguity of the clinical tests for DNC and the many theoretical difficulties DNC defenders face in making their case. Although everything cannot be covered, there are five reasons, from a theoretical perspective, to question her conclusions.

The first concerns Nguyen's belief that the new criteria for death contrast with what she calls "the time-immemorial criteria of total and irreversible cessation of all vital functions, according to which death is declared when respiration has ceased, the heartbeat has stopped, and the body turns grey and cold, followed by other overt signs indicative of a corpse" (3). This suggests that before the era of transplantation, doctors declared death when algor or rigor mortis 
set in. While these conditions are certainly sufficient to declare death, Nguyen's case depends on the stronger claim that they were also necessary. Yet declarations of death have been made on the basis of one's last breath (e.g., Mark 15:37-38). Widely referenced in nineteenth-century medical writing, Benjamin Brodie held that the cessation of breathing alone is a decisive test for extinction of life. ${ }^{1}$ Indeed, the clinical signs of DNC were noted in 1894 by Victor Horsley, who observed that patients who had devastating neurological injuries died from respiratory failure, "not, as so often surmised, from failure of the heart." ${ }^{2}$ Earlier, Charles Hilton Fagge reported in 1886 that patients with brain tumors often die "by cessation of breathing, the heart continuing to beat for some little time." He reports finding a patient "dead, except that a slight flickering of the pulse was still perceptible." ${ }^{3}$ While one could argue that these declarations were erroneous, one cannot argue they were made on criteria that departed from some time-immemorial standard that required sure signs of putrefaction.

Second, DNC defenders are not logically required to view the brain as the supreme integrator of the body. They need only to affirm that the brain plays an indispensable role in the tripartite vital network of brain, lungs, and heart in the continuing existence of the postnatal human organism (e.g., Paul Ramsey's position). ${ }^{4}$ Since the brain is irreplaceable in performing the postnatal human's vital work-maintaining its openness to the world, sensing what it needs (oxygen), and driving the process to get it (inhaling oxygen) - it is plausible to believe that total brain failure marks the end of a human organism as a whole. ${ }^{5}$ While it is right to question the claim that the brain is the sole integrator of the human life form, we should avoid downgrading it so as to think that postnatal humans can live without it and that "the loss of respiratory drive can be substituted by the ventilator" (403). Only the mechanics of airflow can be substituted.

Third, Nguyen's understanding of the decapitation thought experiment, which is meant to show that there would be no fundamental difference between a braindead body and a headless body if they were both artificially maintained, is confused. It need not establish that the headless body is biologically dead or that the brainless body is not an organism as a whole as opposed to a mutilated organism (77). It only needs to show that the headless body is not identity preserving, that the signs of life in a headless body cannot be taken as signs of survival, and that a headless body has taken on a new form that can be identified only as remains. Heuristically speaking, then, the same may be true if a surgeon removed all of the contents of a person's skull or if a person's brain suffered total infarction that obliterated not only his capacity for consciousness but also his drive to breath.

Fourth, despite Nguyen's claims to the contrary, Thomistic metaphysics permits death to mark a substantial change in the nature of the organism without implying a phenomenal change from warm to cold flesh. From an empirical point of view, it may be odd to suppose that a human organism is there one minute and is instantaneously replaced with a numerically different nonhuman organism the next, but the doctrine of virtual presence allows this. Any residual life signs of somatic integration can be the work of a different nonhuman organism after death that was virtually present in the body before death. The time it takes to degrade is irrelevant.

Fifth, while extensively citing the work of Alan Shewmon in support of her views, Nguyen fails to consider three papers in which he gives his in-principle endorsement of DCD, especially the one where he says it would be preposterous to start grieving the loss of a loved one at a poorly defined time well beyond asystole. ${ }^{6}$ These omissions are glaring in a project so heavily reliant on his work yet so defiantly opposed to any form of cadaveric organ procurement.

To be sure, her commitment to the absolute norm against intentionally destroying human life is admirable, yet that is not being undermined today for the reasons she gives.

\section{AdAM OMELIANCHUK}

Adam Omelianchuk, $P h D$, is a clinical ethics fellow at the Stanford Center for Biomedical Ethics in California. 


\section{Notes}

1. The earliest reference I found in English is Julia de Fontenelle, "Médico-légales sur l'incertitude des signes de la mort; les dangers des inhumations précipitées; les moyens de constater les décès et de rappeler à la vie ceux qui sont en état de mort apparente," reprinted in Quarterly Review 85 (June-September 1849), 371.

2. Victor Horsley, "On the Mode of Death in Cerebral Compression, and Its Prevention," Quarterly Medical Journal 2.4 (1894), 305.

3. Charles Hilton Fagge, The Principles and Practice of Medicine, vol. 1 (London: J. and A. Churchill, 1886), 613, emphasis original.

4. Paul Ramsey, The Patient as Person: Explorations in Medical Ethics (New Haven, CT: Yale University Press, 1970), 66-68.
5. President's Council on Bioethics, Controversies in the Determination of Death (Washington, DC: PCBE, 2008), 58-67.

6. D. Alan Shewmon, "Constructing the Death Elephant: A Synthetic Paradigm Shift for the Definition, Criteria, and Tests for Death," Journal of Medicine and Philosophy 35.3 (June 2010): 256-298, doi: 10.1093/jmp/jhq022; D. Alan Shewmon, "The Dead Donor Rule: Lessons from Linguistics," Kennedy Institute of Ethics Journal 14.3 (September 2004): 277-300, doi: 10.1353/ken.2004.0033; and D. Alan Shewmon and Elisabeth Seitz Shewmon, "The Semiotics of Death and Its Medical Implications," in Brain Death and Disorder of Consciousness, ed. Calixto Machado and D. Alan Shewmon (New York: Springer, 2004), 89-114. 